\title{
Digital Volumetric Imaging: Automated Serial Sectioning and Block Face Imaging for Three Dimensional Reconstruction and Visualization of the
}

\section{Biomaterials Interface}

\author{
Janet L. Cuy, Colleen A. Irvin, Maria T. Sundh and Kip D. Hauch \\ University of Washington Engineered Biomaterials Research Center \\ Dept. of Bioengineering, University of Washington, Box 351750 Seattle, WA 98195-1750 USA
}

Visualization of three-dimensional structure is particularly important in biomaterials research where the integration of biomaterial devices and coatings with adjacent tissues is a paramount goal. Tissue engineering approaches to regenerative medicine and wound healing typically deploy macroporous scaffolds seeded with parenchymal cells, which are then cultured with the goal of recapitulating normal tissue structure. Peri-implant neovascularization as well as vessel formation within engineered tissues are important outcomes to assess.

Three dimensional visualization at the microscale can be accomplished with a variety of techniques including serial sectioning and reconstructuion, confocal and multiphoton microscopy, and microcomputed tomography. Digital Volumetric Imaging is an automated serial sectioning and reconstruction technique that is currently under commercial development [1]. Specimens of up to $4 \mathrm{~mm}$ in dimension are fixed, optionally stained in whole, and then embedded in a standard EM resin that has been modified to be opaque. After curing, embedded specimens are mounted on the DVI instrument, which comprises a rotary diamond blade microtome, an epifluorescence microscope and various translation and imaging hardware. In an automated process, sections are cut and then discarded. After each cut, the block face of the specimen is digitally imaged by epifluorescence creating a series of perfectly registered serial images. The opacity of the embedding media reduces fluorescence signal contributions from out of focus planes. Multiple spectral images (e.g. representing distinct fluorophores) are acquired at each plane. The digital serial images are compiled as a three dimensional data set, which is freely navigable and is rendered as threedimensional reconstructions.

Presented in Figure 1 is a macroporous polymeric scaffold comprised of polyhydroxyethylmethacrylate fabricated using a novel sphere templating technique. These scaffolds are being developed for a variety of biomaterial, wound healing, and tissue engineering applications. DVI imaging reveals the uniformity of pore structure and dimensions, pore throat size, interconnectedness, tortuosity, and overall void fraction. Figure 2 presents a similar scaffold material that has been seeded with $\mathrm{C} 2 \mathrm{C} 12$ myoblasts. The distribution, expansion and organization of the cells throughout the porous scaffold are seen. Figure 3 is a DVI reconstruction of intact mouse ventricular myocardium. Myofibrillar organization is seen, along with clear visualization of blood vessels and lumens. To further visualize vasculature in native tissue, the cardiac vessels of a rat were filled through retrograde perfusion with Mercox 2B-CL, and a tissue specimen containing a portion of a coronary vessel cast and the epicardium was obtained. Figure 4 shows the vessel and surrounding fine structure (some distension, an artifact of the casting procedure, is visible). 
The DVI instrument is an improved and automated implementation of time-honored serial sectioning and reconstruction concepts. These examples show the utilization of this powerful new imaging tool in biomaterials and tissue engineering research. Future work will expand on these applications, as well as use of the instrument to assess tissue device integration, facilitated angiogenesis, and to track the fate of exogenous cells in cell transplantation research.

[1] www.microsciencegroup.com.

[2] This work was supported by University of Washington Engineered Biomaterials (UWEB), a NSF Engineering Research Center (NSF EC 9529161) and by BioEngineered Allogeneic Tissue (BEAT) a NIH Bioengineering Research Partnership (R24 HL64387) focused on the tissue engineering of cardiac muscle.

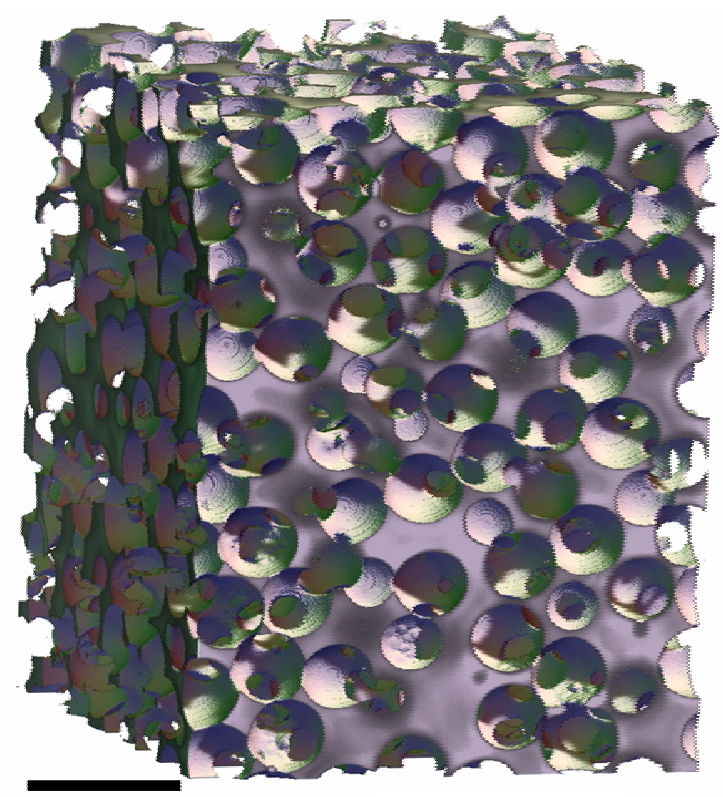

$200 \mu \mathrm{m}$
Figure 1

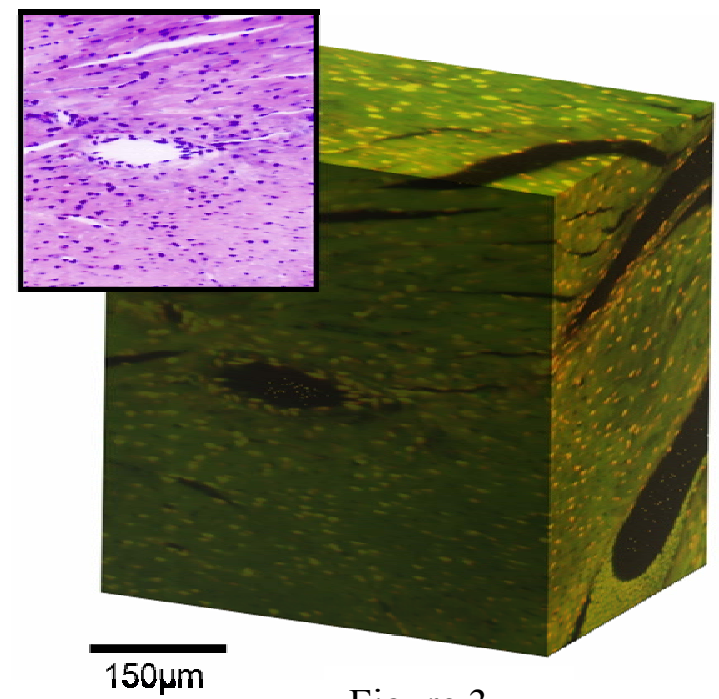

Figure 3

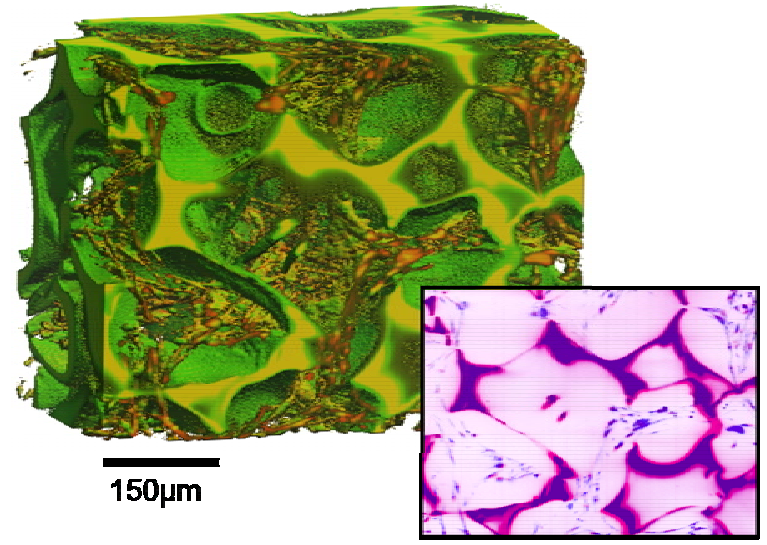

Figure 2

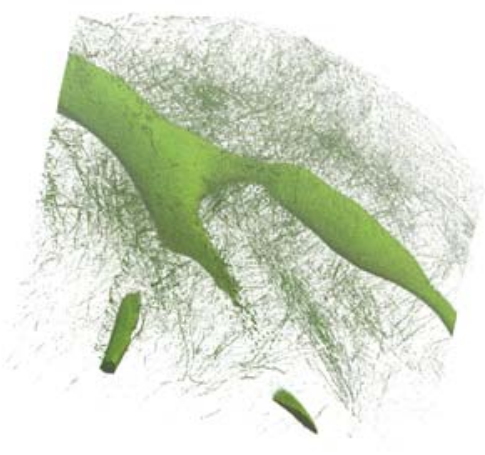

Figure 4 